\title{
Effects of Periodontal Disease and Periodontal Treatment on the Lipid Profile: A Sri Lankan Study
}

\author{
${ }^{1}$ A Tilakaratne, ${ }^{2}$ WKL Perera, ${ }^{3}$ EAAP Amaratunga, ${ }^{4} \mathrm{R}$ Sivakanesan
}

\begin{abstract}
Aims: To compare the blood lipid levels of periodontitis group (of different severities) with a nonperiodontitis group and to evaluate any influence of periodontal treatment in altering blood lipid levels.

Materials and methods: The study sample consisted of 83 patients with periodontitis in the "test" group and a matched "control" group of 40 , free of periodontitis according to predetermined selection criteria. All subjects underwent periodontal assessments and blood lipid estimation prior to treatment planning, following which they received active periodontal treatment/oral prophylaxis. Twelve weeks after treatment, periodontal reevaluations were carried out and blood lipids were reassessed for pre- and posttreatment comparisons.
\end{abstract}

Results: Increases in lipid levels were observed in the periodontitis group compared to the nonperiodontitis group at baseline. The test group had $5 \%$ higher mean total cholesterol than the control group at pretreatment/preintervention (albeit not significant: Student's t), while low-density lipoprotein cholesterol (LDL-Ch) was $6.6 \%$ higher in the test group than the control group ( $95 \%$ confidence interval). Mean triglycerides (Tri-G) were $5.7 \%$ higher in the control group than the test group, yet without statistical significance. All types of lipids in both the groups had decreased following periodontal therapy, while Tri-G levels demonstrated an opposite effect.

Conclusion: Patients with generalized severe periodontitis had higher LDL-Ch values than those with generalized mildmoderate periodontitis. Periodontal care was effective in lowering unfavorable serum lipids. Prompt control of periodontal inflammation would minimize development of deep periodontal infection and cardiovascular risk.

Keywords: Cardiovascular disease, Cardiovascular disease risk, Lipid profile, Low-density lipoprotein cholesterol, Periodontal treatment, Periodontitis.

How to cite this article: Tilakaratne A, Perera WKL, Amaratunga EAAP, Sivakanesan R. Effects of Periodontal Disease and Periodontal Treatment on the Lipid Profile: A Sri Lankan Study. Int J Experiment Dent Sci 2016;5(2):127-132.

\footnotetext{
${ }^{1}$ Professor, ${ }^{2}$ Postgraduate Trainee, ${ }^{3}$ Medical Officer, ${ }^{4}$ Senior Professor

${ }^{1}$ Department of Oral Medicine and Periodontology, Faculty of Dental Sciences, University of Peradeniya, Sri Lanka

${ }^{2}$ Division of Periodontology, Faculty of Dental Sciences University of Peradeniya, Sri Lanka

${ }^{3}$ Base Hospital - Matale, Sri Lanka

${ }^{4}$ Department of Biochemistry, Faculty of Medicine, University of Peradeniya, Sri Lanka

Corresponding Author: A Tilakaratne, Professor, Department of Oral Medicine and Periodontology, Faculty of Dental Sciences University of Peradeniya, Sri Lanka, Phone: 0094812397450 e-mail: tlkrtn@yahoo.com
}

Source of support: University of Peradeniya Sri Lanka (Research Grant Number RG/2009/40/D).

\section{Conflict of interest: None}

\section{INTRODUCTION}

Hyperlipidemia and periodontitis are highly prevalent chronic diseases in the world population, with periodontal disease being the most prevalent adult oral health problem in Sri Lanka. ${ }^{1}$ Periodontal disease is a chronic inflammatory disease caused by pathogens in plaque biofilms adherent to the tooth and its supporting tissues. The inflammatory response to plaque biofilm leads to destruction of toothsupporting structures. The main organisms implicated in advanced periodontitis are Gram-negative anaerobes, which produce lipopolysaccharides (LPS). Although pathogen-derived LPS triggers acute-phase reactions, the occurrence and severity of periodontal disease also depend on many exposure factors, both local and systemic/genetic risk factors including immunological abnormalities. ${ }^{2}$ The role of host immune response in periodontal infection has been reviewed and a dysregulated host response has been shown in more aggressive periodontitis. ${ }^{3}$

Dyslipidemia is diagnosed when low-density lipoprotein (LDL-Ch) cholesterol is raised, high-density lipoprotein (HDL-Ch) is low, and triglyceride (Tri-G) levels are high, which are associated with an increased risk of coronary heart disease (CHD). Literature reveals a bidirectional relationship between periodontitis and atherosclerotic cardiovascular disease (CVD), diabetes mellitus (DM), ${ }^{4,5}$ and dyslipidemia. Dyslipidemia is an established causative factor for atherosclerosis and $\mathrm{CVD}^{6}$ thus explaining a possible multidirectional relationship among each other. Multivariate logistic regression analysis has revealed significant correlations between dyslipidemia and periodontitis. ${ }^{7}$ It is known that pro-inflammatory cytokines, such as TNF- $\alpha$ and IL-6, which are produced in relation to periodontal infection, play a significant role in promoting dyslipidemia. Triglycerides, LDL, and glucose have also been significantly elevated in subjects with severe periodontitis and $\mathrm{CHD}^{8}{ }^{8}$

As evident in literature, systemic inflammation could alter blood lipid metabolism. Therefore, it is plausible that periodontitis, which is a deep periodontal infection, could lead to an altered blood lipid metabolism. Clinical trials indicate that treatment of periodontitis could result 
in an improvement in dyslipidemic status. In light of the above, the aims of this research were to compare blood lipid levels of "periodontitis patients" with a "control group" (free of periodontitis); and to assess any possible relationship between the severity of periodontitis and blood lipids as well as to evaluate any influence of periodontal interventions in altering blood lipids.

\section{MATERIALS AND METHODS}

\section{Study Population}

This was an experimental study design. The study population was purposively selected and it consisted of all suitable subjects who attended the Study Centre during the recruitment period. The study population consisted of a "test" group $(\mathrm{n}=83)$ and a "control" group $(n=40)$ matched in relation to age $(35-50 \pm 5$ years $)$, socioeconomic parameters, dietary habits, and oral hygiene practices. The study was carried out in the Division of Periodontology, Faculty of Dental Sciences, University of Peradeniya, Sri Lanka, following approval of Faculty Research Ethics Review Committee. All participants gave informed written consent, and they were selected according to the inclusion criteria of the study. These criteria were: (1) No history of known confounding systemic conditions, such as hyperlipidemia, DM, ischemic heart disease, obesity, or pregnancy; (2) no family history of dyslipidemia; (3) no evidence of infection within the preceding 3 months; (4) not on lipid-lowering medication or other medication; (5) not undergone periodontal treatment for the past 6 months; and (6) never smoked. As DM is an increasingly common perplexing factor, all subjects were screened by conforming to fasting blood sugar (FBS) assessments.

\section{Sample Size}

Sample sizes of 100 for the "test" group and 100 for the "control" group were originally projected. This was after going through the "New Patient Register" maintained in the clinic for the preceding 2 years, which directed the possibility of recruiting the above numbers approximately.

The originally recruited numbers for test and control groups were 112 and 78 respectively. After screening for $\mathrm{DM}$, all subjects underwent pretreatment (pre-Rx) blood lipid assessment, followed by periodontal evaluations and treatment. However, only 83 subjects (out of 112) in the test group and 40 (out of 78) in the control group were available/qualified for the 12-week posttreatment (post$\mathrm{Rx}$ ) evaluations, hence the reason for including test $=83$ and control $=40$ in the analysis of results to compare pre-Rx and 12-weeks' post-Rx data.

Since dropout of patients was a drawback in the study, it was not possible to sustain an adequate sample size beyond 12 weeks for the intended long-term post-Rx evaluations.

\section{Periodontal Assessments}

All subjects underwent a full-mouth clinical periodontal assessment (FMCPA) in order to rationalize the presence/ absence of periodontitis. Full-mouth clinical periodontal assessment was performed at six sites per tooth, using a William's periodontal probe. Periodontal parameters included percentages of plaque, bleeding on probing $(\mathrm{BOP})$, probing depths $(\mathrm{PD})$ in $\mathrm{mm}$, gingival recession (in $\mathrm{mm}$ ), loss of attachment (LOA), furcation involvement, and grading of tooth mobility. In the presence of gingival recession, LOA was obtained by adding recession and PD measurements. Where there was no recession, LOA was obtained by subtracting the "distance from gingival margin to cemento-enamel junction" from the "total PD."

The test group included patients with periodontitis according to the FMCPA and established diagnostic criteria followed in the Unit. ${ }^{9}$ The control group included subjects free of periodontitis (adjudged by the FMCPA) with slight marginal gingival inflammation who attended for dental consultations, followed by oral prophylaxis.

After FMCPA, radiographic evaluations were performed on the test group in order to decide on the definitive treatment plan. Periodontal diagnoses were made according to the severity and distribution of periodontitis. Severity was categorized based on the guidelines denoting the clinical attachment levels and radiographic evidence of bone levels. ${ }^{9}$ Thus, the categorization included four distinct groups, namely, "Localized PeriodontitisMild-Moderate," "Localized Periodontitis-Severe," "Generalized Periodontitis-Mild-Moderate," and "Generalized Periodontitis-Severe." This categorization reflected the overall magnitude and disease burden where "Localized Periodontitis-Mild-Moderate" was considered as the least severe category and "Generalized Periodontitis-Severe" as the most severe category. The guidelines for severity grading were: (1) Mild-Moderate $=$ LOA of less than $5 \mathrm{~mm}$ (clinically) and loss of bone up to half the root length radiographically; (2) severe $=\mathrm{LOA} 5+\mathrm{mm}$ and loss of bone greater than half the root length with or without furcation involvement. The distribution was categorized as "localized" if the total sites involved were less than $30 \%$ of all the examined sites, while more than $30 \%$ site involvement was considered as of "generalized" category.

All periodontal assessments were carried out by a well-trained practitioner, following intraexaminer calibration and informed consent of the subjects.

\section{Laboratory Assessments}

All subjects were first screened to exclude DM by conforming to FBS measurement. Within 3 days following 
Table 1: Comparison of lipid and triglyceride levels in the test and control groups before (pre-) and after (post-) treatment/intervention

\begin{tabular}{|c|c|c|c|c|c|c|c|c|c|}
\hline \multirow{2}{*}{$\frac{\text { Groups }}{\text { Control }}$} & & \multicolumn{2}{|c|}{ TOTAL-Ch (mg/100 mL) } & \multicolumn{2}{|c|}{$H D L-C h(m g / 100 m L)$} & \multicolumn{2}{|c|}{ LDL-Ch (mg/100 mL) } & \multicolumn{2}{|c|}{$\begin{array}{l}\text { Triglyceride } \\
(\mathrm{mg} / 100 \mathrm{~mL})\end{array}$} \\
\hline & & Pre-I & Post-I & Pre-I & Post-I & Pre-I & Post-I & Pre-I & Post-I \\
\hline & Mean & 205.71 & 195.41 & 42.62 & 38.08 & 137.18 & 127.74 & 142.67 & 149.11 \\
\hline & $\mathrm{N}$ & 40 & 40 & 40 & 40 & 40 & 40 & 40 & 40 \\
\hline & Standard deviation & 38.8025 & 40.2500 & 10.9400 & 13.1368 & 30.9354 & 28.6228 & 62.466 & 61.967 \\
\hline \multirow[t]{4}{*}{ Test } & & Pre-Rx & Post-Rx & Pre-Rx & Post-Rx & Pre-Rx & Post-Rx & Pre-Rx & Post-Rx \\
\hline & Mean & 215.53 & 204.67 & 43.66 & 39.74 & 146.31 & 137.515 & 134.99 & 136.79 \\
\hline & $\mathrm{N}$ & 83 & 83 & 83 & 83 & 83 & 83 & 83 & 83 \\
\hline & Standard deviation & 45.2613 & 38.7155 & 11.4660 & 10.3018 & 39.7814 & 34.4733 & 65.082 & 56.724 \\
\hline \multicolumn{10}{|c|}{$\begin{array}{l}\text { Statistical } \\
\text { significance }\end{array}$} \\
\hline $\begin{array}{l}\text { (Student's } \\
\text { t-test) }\end{array}$ & & $t=1.975$ & $t=1.226$ & $t=0.833$ & $t=0.762$ & ${ }^{*} t=2.09$ & $t=1.553$ & $t=1.075$ & $t=1.09$ \\
\hline
\end{tabular}

recruitment of subjects, prior to periodontal treatment or any intervention, their lipid profile (Table 1) was assessed. Venous blood $(5 \mathrm{~mL})$ was obtained from each subject after 12 hours of fasting. Blood samples were obtained in the Haematology Laboratory in the Study Centre and then transported to the Department of Biochemistry, Faculty of Medicine, University of Peradeniya, Sri Lanka, for assessment. Plasma lipids and triglyceride levels were determined by routine enzymatic methods. [The following cut-off points were considered as pathological according to the laboratory's recommendations: Total cholesterol (TOTAL-Ch) $>230 \mathrm{mg} / \mathrm{dL}$; LDL cholesterol > $160 \mathrm{mg} / \mathrm{dL}$; HDL cholesterol < $45 \mathrm{mg} / \mathrm{dL}$; Total cholesterol/HDL ratio > 5.5]. ${ }^{10}$

\section{Experimental Protocol}

All patients in the test group underwent treatment for periodontitis, while the control group received routine oral prophylaxis. Both groups were followed up for 3 months' periodontal maintenance care.

The periodontal treatment protocol for the test group included sessions of plaque control methods, elimination of plaque-retentive areas, systematic quadrant-wise scaling and closed root surface debridement (SC and RSD), and stringent maintenance care. Following active treatment, patient compliance was reviewed at 2-weekly intervals during maintenance phase for 12 weeks. Similarly, the control group was also followed up for the maintenance phase for 12 weeks at 2-weekly intervals, after oral prophylaxis.

Twelve weeks following interventions, periodontal reevaluations were performed with a FMCPA, and the same parameters (BOP, $\mathrm{PD}, \mathrm{LOA}$, mobility) and plaque scores were reassessed. Blood lipid profiles were also assessed at 12 weeks following treatment/oral prophylaxis.

\section{Statistical Design}

The data were processed and the mean values and standard deviations of TOTAL-Ch, LDL-Ch, HDL-Ch, and Tri-Gs for test and control groups at pretreatment and posttreatment were tabulated. Paired sample correlations were analyzed and the Student's t-test was applied to denote any significance of the results. Accordingly, a $\mathrm{t}$-value of $\geq 1.99$ in the $\mathrm{t}$-table was denoted as the cut-off value for a significant result at $95 \%$ confidence interval.

In order to test for a reliably matching control group for test group comparisons, socioeconomic status (SES) was established by obtaining a composite index for their occupation and education, which has previously been validated as a method to categorize SES in a study carried out in Sri Lanka. ${ }^{11}$ Thus, the information on occupation and education was amalgamated to represent the SES of subjects where a scale (1-10) from correspondence analysis was obtained and the subjects were categorized into three groups (Low-, Middle-, and High-Socioeconomic groups). Only the subjects belonging to "middle SES" category were included in the analysis. Similarly, matching for dietary habits was carried out cautiously only to include those who could be considered as "conformists," of typical Sri Lankan diet. With the corresponding analyses, subjects were categorized into three groups based on their overall consumption of fats, sugars, and carbohydrates (FSC). The categories were Low-, Moderate- and High-FSC consumers. Only the "moderate FSC consumers" were included in the analysis.

\section{RESULTS}

Mean values of TOTAL-Ch, HDL-Ch, LDL-Ch, and Tri-G in $\mathrm{mg} / 100 \mathrm{~mL}$ for control and test groups were compared at preintervention/pretreatment (Table 1). Accordingly, 
the test group had 5\% higher mean TOTAL-Ch than the control group prior to interventions. Nevertheless, this increase was not statistically significant. Yet, neither the test nor the control group revealed abnormal values of TOTAL-Ch. Similarly, LDL-Ch values of test and control groups were within the normal range. However, it was $6.6 \%$ higher in the test group when compared to the control, and the difference was statistically significant at $95 \%$ confidence interval $(t=2.09)$. A similar trend was apparent with regard to HDL-Ch in both the groups. Although HDL-Ch values were within the normal range, the value for the test group was $2.6 \%$ higher than that of the control, though not statistically significant. In contrast, the mean value of Tri-G was $5.7 \%$ higher in the control group than the test group, without any statistical significance.

Notably, 12 weeks after interventions/treatment, it was observed that TOTAL-Ch, HDL-Ch, and LDL-Ch levels had reduced in both the groups when compared with their respective pretreatment values, while the Tri-G levels have shown an opposite effect (increase) in both the groups.

Table 2 depicts paired sample correlations for the control group. Accordingly, the reductions in TOTAL-Ch, HDL-Ch, LDL-Ch, and TOTAL-Ch/HDL ratio were statistically significant at $95 \%$ confidence interval, although the difference in mean Tri-G was not statistically significant.

Table 3 represents paired sample correlations for the test group before and after treatment. Here too, a similar

Table 2: Paired sample correlations for the control group

\begin{tabular}{|c|c|c|c|c|c|c|c|c|}
\hline \multicolumn{9}{|c|}{$\begin{array}{l}\text { Paired samples test (control group) } \\
\text { Paired differences }\end{array}$} \\
\hline & $\begin{array}{l}\text { Mean } \\
(\mathrm{mg} / 100 \mathrm{~mL})\end{array}$ & $\begin{array}{l}\text { Std } \\
\text { deviation }\end{array}$ & Std error mean & $\begin{array}{l}95 \% \text { confidence } \\
\text { interval of the } \\
\text { difference }\end{array}$ & & & & \\
\hline & & & & Lower & Upper & $T$ & $d f$ & (2-tailed) \\
\hline Pair 1: Pre-TOTAL-Ch & 10.30000 & 21.34348 & 3.37470 & 3.47402 & 17.12598 & 3.052 & 39 & $0.004^{*}$ \\
\hline \multicolumn{9}{|l|}{ Post-TOTAL-Ch } \\
\hline Pair 2: Pre-HDL-Ch & 4.53560 & 7.53329 & 1.19112 & 2.12634 & 6.94487 & 3.808 & 39 & $0.000^{*}$ \\
\hline \multicolumn{9}{|l|}{ Post-HDL-Ch } \\
\hline Pair 3: Pre-LDL-Ch & 9.44500 & 9.16680 & 1.44940 & 6.51331 & 12.37669 & 6.516 & 39 & $0.000^{*}$ \\
\hline \multicolumn{9}{|l|}{ Post-LDL-Ch } \\
\hline \multicolumn{9}{|c|}{ Pair 4: Pre-TOTAL-Ch_HDL-Ch } \\
\hline \multicolumn{9}{|c|}{ Post-TOTAL-Ch_HDL-Ch } \\
\hline & -0.39667 & 0.73672 & 0.11649 & -0.63229 & -0.16106 & 3.405 & 39 & $0.002^{*}$ \\
\hline Pair 5: Pre-Tri-G & -6.43250 & 47.05132 & 7.43947 & -21.48024 & 8.61524 & -0.865 & 39 & 0.393 \\
\hline Post-Tri-G & & & & & & & & \\
\hline
\end{tabular}

Std deviation/Std error: Standard deviation/Standard error; T: t value; Pre-: Before intervention; Post-: After intervention; Ch: Cholesterol; Tri-G: Triglycerides; *Statistically significant. According to the t table, the t-value should be $\geq 2.02$ to be significant at least at $95 \%$ confidence level $(n=40)$

Table 3: Paired sample correlations for the test group

\begin{tabular}{|c|c|c|c|c|c|c|c|c|}
\hline \multicolumn{9}{|c|}{$\begin{array}{c}\text { Paired samples test (test group) } \\
\text { Paired differences }\end{array}$} \\
\hline & $\begin{array}{l}\text { Mean } \\
(\mathrm{mg} / 100 \mathrm{~mL})\end{array}$ & $\begin{array}{l}\text { Std } \\
\text { deviation }\end{array}$ & $\begin{array}{l}\text { Std error } \\
\text { mean }\end{array}$ & $\begin{array}{l}95 \% \text { confidence } \\
\text { interval of the } \\
\text { difference }\end{array}$ & & & & \\
\hline & & & & Lower & Upper & $T$ & $d f$ & $\begin{array}{l}\text { Significance } \\
\text { (2-tailed) }\end{array}$ \\
\hline Pair 1: Pre-TOTAL-Ch & 10.85542 & 22.26980 & 2.44443 & 5.99267 & 15.71817 & 4.441 & 82 & $0.000^{*}$ \\
\hline \multicolumn{9}{|l|}{ Post-TOTAL-Ch } \\
\hline Pair 2: Pre-HDL-Ch & 3.92202 & 7.40484 & 0.81279 & 2.30513 & 5.53891 & 4.825 & 82 & $0.000^{*}$ \\
\hline \multicolumn{9}{|l|}{ Post-HDL-Ch } \\
\hline Pair 3: Pre-LDL-Ch & 8.79398 & 13.22243 & 1.45135 & 5.90678 & 11.68117 & 6.059 & 82 & $0.000^{*}$ \\
\hline \multicolumn{9}{|l|}{ Post-LDL-Ch } \\
\hline \multicolumn{9}{|c|}{ Pair 4: Pre-TOTAL-Ch_HDL-Ch } \\
\hline Post-TOTAL-Ch_HDL-Ch & -0.17333 & 0.68339 & 0.07501 & -0.32255 & -0.02411 & -2.311 & 82 & $0.023^{*}$ \\
\hline \multicolumn{9}{|l|}{ Pair 5 Pre-Tri-G } \\
\hline Post-Tri-G & -1.79711 & 31.73781 & 3.48368 & -8.72725 & 5.13304 & -0.516 & 82 & 0.607 \\
\hline
\end{tabular}

Std deviation/Std error: Standard deviation/standard error; T: t value; Pre-: Before treatment; Post-: After treatment; Ch: Cholesterol; Tri-G: Triglycerides; *Statistically significant at least at $95 \%$ confidence level 
Effects of Periodontal Disease and Periodontal Treatment on the Lipid Profile: A Sri Lankan Study

Table 4: Comparison of the severity of periodontitis and lipid levels in the test group

\begin{tabular}{|c|c|c|c|c|c|}
\hline Periodontitis-severity category & TOTAL-Ch & $H D L-C h$ & $L D L-C h$ & TOTAL-Ch/HDL-Ch & Tri-G \\
\hline $\begin{array}{l}\text { Localized Periodontitis } \\
\text { ("Mild-Moderate" vs "Severe") }\end{array}$ & & & & & \\
\hline $\begin{array}{l}T \\
t \geq 2.08, N=23\end{array}$ & 0.069891439 & -0.889925013 & -0.040979256 & -0.015148054 & 0.299648671 \\
\hline $\begin{array}{l}\text { Generalized Periodontitis } \\
\text { ("Mild-Moderate" vs "Severe") }\end{array}$ & & & & & \\
\hline $\begin{array}{l}T \\
t \geq 2.00, N=58\end{array}$ & -1.408226193 & 1.321395 & $2.433667261^{*}$ & -0.49217 & 0.243892 \\
\hline
\end{tabular}

Ch: Cholesterol; T: $t$ value; $\mathrm{N}$ : Number; ${ }^{*}$ Statistically significant at $95 \%$ confidence; $\mathrm{t} \geq$ value for a significant result

reduction was evident where the differences between pre- and posttreatment values for TOTAL-Ch, HDL-Ch, LDL-Ch, and TOTAL-Ch/HDL-Ch ratio were statistically significant at $95 \%$ confidence interval. However, the difference between pre- and post-Tri-G levels was not statistically significant.

Table 4 gives $t$ values and their significance based on the mean lipid and Tri-G values obtained for patients categorized as "Localized Periodontitis" or "Generalized Periodontitis" and severity categorization as "Mild-Moderate" or "Severe" periodontitis. Thus, the analysis was based on the mean values for four distinct groups, namely: (1) Localized Periodontitis-Mild to Moderate, (2) Localized Periodontitis-Severe, (3) Generalized Periodontitis-Mild to Moderate, and (4) Generalized Periodontitis-Severe.

The previous categorization reflects the overall magnitude of disease, where "Localized Periodontitis-Mild to Moderate" was considered as the "least-severe" category and "Generalized Periodontitis-Severe" as "most severe."

The $t$ values obtained for comparison of TOTAL-Ch, HDL, LDL, TOTAL-Ch/HDL ratio and Tri-G between "Localized Periodontitis-Mild-Moderate" group and "Localized Periodontitis-Severe" group were less than what is required $(\geq 2.08)$ for a significant result. In other words, with localized disease, lipid and Tri-G levels have not been significantly different between the two severity categories. However, the $t$ values obtained for the same comparisons in "Generalized Periodontitis-Mild-Moderate" category and "Generalized Periodontitis-Severe" category showed a significant result, only for the LDL-Ch levels ( $\mathrm{t}>2.00)$. This indicates that the patients affected with "severe" generalized periodontitis had significantly higher LDL-Ch level when compared to those having "mild-moderate" generalized periodontitis.

\section{DISCUSSION}

In the present study, the baseline mean values of TOTAL$\mathrm{Ch}$ and LDL-Ch were higher in the test group than those in the control group. However, only the difference between LDL-Ch of the two groups showed significance statistically. Yet, the aforementioned result is in agreement with the trend already reported globally to explain the association between hyperlipidemia and periodontitis. This supports the notion that chronic infections, such as periodontitis could modify the serum lipid profile, promoting unfavorable lipids in a way that would increase the risk of atherosclerosis. Possible mechanisms linking hyperlipidemia and periodontitis report that hyperlipidemia could induce hyperactivity of leukocytes. A recent pilot study demonstrates a significant dyslipidemic profile and elevated levels of the inflammatory agents C-reactive protein, IL-6, and TNF- $\alpha$ in subjects with CHD and chronic periodontitis compared with those without periodontitis. ${ }^{12}$

However, dyslipidemia is implicated as a multifactorial condition, since numerous factors influence its occurrence in an individual. In this context, lipid profiles of the Sri Lankan study population with periodontitis are noteworthy. There is also evidence to validate the concept that treatment of periodontal disease has beneficial effects on lipid metabolism, with significant improvement in lipid profiles in response to periodontal treatment possibly due to decreased serum levels of pro-inflammatory cytokines. ${ }^{13}$ It is interesting that, in the present study, all types of lipids in both groups were reduced with either periodontal treatment or interventions, while Tri-G levels have shown an opposite effect. While this highlights the importance of periodontal treatment and other therapeutic interventions in lowering the blood lipids, it may also indicate a possible balance maintained between serum lipids and triglycerides, albeit with undefined precision for such a mechanism. As such, a recent research has reported that higher levels of tri-glycerides and lower levels of HDL-Ch were associated with the presence of Treponema denticola in chronic periodontitis, suggesting a possible mechanism that these organisms could reduce anti-atherogenic potency of HDL-Ch. ${ }^{14}$

Even though the mean lipid values in the test and control groups in the present study have not exceeded the accepted normal values, these findings can be extrapolated to reinforce the importance of periodontal therapy and preventive care in bringing about a favorable lipid profile, if it is to be applied to individuals with abnormally altered lipid levels. 
The findings of this study portray the impact of more generalized severe form of periodontitis with a greater inflammatory burden in influencing the rise of the most unfavorable serum lipid, LDL-Ch, in contrast to generalized, but mild-moderate category of periodontitis. It also highlighted that "localized" periodontitis, irrespective of its severity, may not be adequate to elicit significant changes in the lipid profile of such patients. Therefore, it is essential that periodontal inflammation is controlled promptly both by clinicians and by patients, without permitting it to establish in a generalized form, which in turn would lead to adverse systemic consequences, such as heightened cardiovascular risk markers.

It is anticipated that an expanded long-term follow-up study with a larger study sample would enable a more detailed analysis based on sex, age groups, and more specific severity categories of periodontitis. Such an analysis would provide aspects pertaining to specificity and clarity of this association.

\section{CONCLUSION}

Periodontitis is inflammation of the tissues surrounding the tooth affecting the gingiva, periodontal ligaments and the bone and in its severe forms there can be loss of bone that supports the tooth, resulting in the tooth becoming loose and even causing tooth loss. ${ }^{15}$ Resultant tooth loss would not only be a local problem confined to the oral cavity and its function/esthetics, but would have adverse implications on the systemic health.

\section{ACKNOWLEDGMENT}

The authors wish to thank, Mrs W.C. Mohotty and Miss P.M. Perera (Department of Oral Medicine and Periodontology, Faculty of Dental Sciences, Peradeniya, Sri Lanka) for their valuable technical support in carrying out laboratory investigations. Prof. Mena Soory (King's College London Dental Institute, London) for her kind help and valuable comments during the manuscript preparation. Dr. Sanjeewa Kularatne (Griffith University, Australia) for his kind assistance in statistical analysis.

\section{REFERENCES}

1. Ministry of Health. National Oral Health (NOH) Survey of Sri Lanka. Report of the Ministry of Health Colombo; 2003.
2. Cantore S, Mirgaldi R, Ballini A, Coscia MF, Scacco S, Papa F, Inchingolo F, Dipalma G, De Vito D. Cytokine gene polymorphisms associate with microbiogical agents in periodontal disease: our experience. Int J Med Sci 2014 May 1; 11(7):674-679.

3. Schmidt J, Jentsch H, Stingu CS, Sack U. General immune status and oral microbiology in patients with different forms of periodontitis and healthy control subjects. PLoS One 2014 Oct 9;9(10):e109187.

4. Borgnakke WS, Ylostalo PV, Taylor GW, Genco RJ. Effects of periodontal disease on diabetes: systematic review of epidemiological observational evidence. J Clin Periodontol 2013 Apr;40 (Suppl 4):S135-S152.

5. Chapple IL, Genco RJ. Diabetes and periodontal diseases: consensus report on the joint EFP/AAP workshop on periodontitis and systemic diseases. J Clin Periodontol 2013 Apr;40 (Suppl 14):S106-S112.

6. Carramolino-Cuéllar E, Tomás I, Jiménez-Soriano Y. Relationship between the oral cavity and cardiovascular diseases and metabolic syndrome. Med Oral Patol Oral Cir Bucal 2014 May;19(3):e289-e294.

7. Lee JB, Yi HY, Bae KH. The association between periodontitis and dyslipidemia based on the Fourth Korea National Health and Nutrition Examination Survey. J Clin Periodontol 2013 May;40(5):437-442.

8. Flores MF, Montenegro MM, Furtado MV, Polanczyk CA, Rösing CK, Haas AN. Periodontal status affects C-reactive protein and lipids in patients with stable heart disease from a tertiary care cardiovascular clinic. J Periodontol 2014 Apr;85(4):545-553.

9. Chapple ILC, Gilbert AD. Understanding periodontal diseases: assessment and diagnostic procedures in practice. London: Quintessence Publishing Co. Ltd; 2002. p. 111-146.

10. Wood D. European and American recommendations for coronary heart disease prevention. Eur Heart J 1998 Feb;19 (Suppl A):A12-A19.

11. Amarasinghe HK, Johnson NW, Lalloo R, Kumaraarachchi M, Warnakulasuriya S. Deviation and validation of a risk-factor model for detection of oral potentially malignant disorders in populations with high prevalence. Br J Cancer $2010 \mathrm{Jul}$ 27;103(3):303-309.

12. Tang $\mathrm{K}$, Lin $\mathrm{M}, \mathrm{Wu} \mathrm{Y}, \mathrm{Yan} \mathrm{F}$. Alteration in serum lipid and inflammatory cytokine profiles in patients with coronary heart disease and chronic periodontitis: a pilot study. J Int Med Res 2011;39(1):238-248.

13. Altay U, Gurgan CA, Agbaht K. Changes in inflammatory and metabolic parameters after periodontal treatment in patients with and without obesity. J Periodontol 2013 Jan;84(1):13-23.

14. Ardila CM, Olarte-Sossa M, Ariza-Garces AA. Association between the presence of Treponema denticola and reduced levels of antiatherogenic high density lipoprotein in periodontitis. Quintessence Int 2015 Mar;46(3):207-215.

15. Saini R. A Prospective Experimental Comparative Study on the Clinical Effects of Calculus Dissolution based Oral Rinse in Gingivitis Patients. Int J Experiment Dent Sci 2015;4(1):33-39. 